\title{
Influence of mode competition and external wave frequency modulation on gyrotron frequency locking
}

\author{
Yu.V. Novozhilova, V.L. Bakunin, A.V. Chirkov, Yu.M. Guznov, G.G. Denisov, A.P. Fokin, \\ A.S. Shevchenko, N.I. Zaitsev, S.A. Zapevalov
}

IAP RAS, Nizhny Novgorod, Russia, julia.novozhilova2009@yandex.ru

\section{Introduction}

For a number of practical applications, such as plasma diagnostics or high-resolution spectroscopy, and in prospect for development of the coherently radiating gyrotron complex for large CTS setups, it is important to have a source of radiation with a highly stable frequency. Locking of gyrotron oscillation frequency by an external monochromatic wave seems to be a promising way for obtaining such source. Although in the recent decades influence of the external monochromatic signal on the gyrotron operation was studied in a number of publications [1-6], specific designs of megawatt power level gyrotrons have not been discussed. Study of frequency stabilization by a wave coming into the cavity from the external tract became of particular interest since the development of the quasi-optical converter which allows to transform a large part of wave coming from the output section into the gyrotron operating mode at the Institute of Applied Physics RAS (IAP RAS) [7]. The aim of this paper is to determine the optimal parameters of the gyrotron and of the external signal for efficient single-mode generation in the multimode gyrotron at a stable frequency. Accordingly, we consider a number of problems of multimode gyrotron frequency locking by an external signal.

\section{Influence of the mode competition}

\section{on the locking regime in the multimode gyrotron}

We study frequency locking of a multimode gyrotron by an external monochromatic signal with a frequency close to the eigenfrequency of the operating mode. We use the approximation of fixed longitudinal structure of the field, which is valid at sufficiently high Q-factor of gyrotron cavity. This approximation allows us to study competition of several modes, including non-equidistant modes with frequency detuning comparable to the cyclotron absorption linewidth. Locking was simulated based on the example of a megawatt gyrotron prototype for ITER developed at the IAP RAS operating at TE28.12 mode at a frequency of $170 \mathrm{GHz}$ with a quality factor of 1370; accelerating voltage at the end of switching stage was about $90-100 \mathrm{keV}$ with a current of 50-60 A. Scenario of electron beam parameters switching was taken similar to the real one, which is used to achieve high efficiency.

Locking zones, i.e., regions where the operating mode is excited at the frequency of the external signal, were determined on the planes of various parameters: current and detuning $\Delta=\left(2 / \beta_{\perp}^{2}\right)\left(1-\omega_{H} / \omega_{0}\right)$ (dimensionless detuning between gyro-frequency and eigen frequency of the operating mode and current), as well as detuning $\Delta$ and frequency of the external signal (Fig. 1, 2 ). We show that the width of the locking zones and max- imum achievable current in the multimode gyrotron demonstrate non-monotonous dependence on the the external signal power, which can be explained by nonlinear mode competition (Fig. 1b). When the operating mode frequency of a multimode gyrotron is locked, much higher efficiency can be achieved and the frequency tuning band increases by several times in comparison to a free gyrotron (Fig. 1, 2).
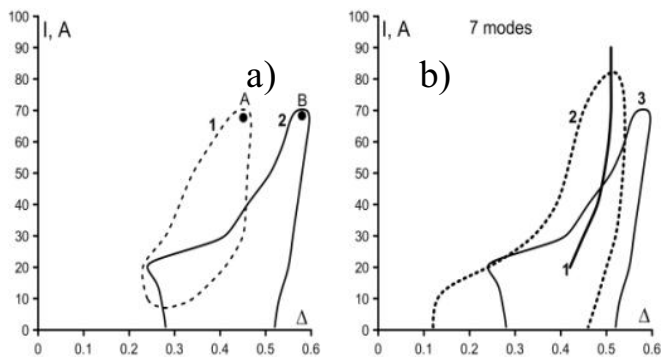

Fig. 1. Region of single-frequency generation at $\mathrm{TE}_{28.12}$ operating mode in free gyrotron (curve 1, Fig. 1a) and locking zones (curve 2, Fig. 1a and Fig. 1b) in 7-mode gyrotron (parasitic modes $\mathrm{TE}_{26.12}, \mathrm{TE}_{27.12}, \mathrm{TE}_{29.12}, \mathrm{TE}_{30.12}, \mathrm{TE}_{-25.13}, \mathrm{TE}_{-26.13}$ ). 1a) external signal power is $5 \%$ of radiation power, maximum efficiency is achieved for free gyrotron at point A (63\%) and for locked gyrotron at B $(82 \%)$. Locking zones in Fig. $1 \mathrm{~b}$ correspond to different values of external signal power: 1) $0.01 \%$ of radiation power, 2) $0.5 \%, 3) 5 \%$

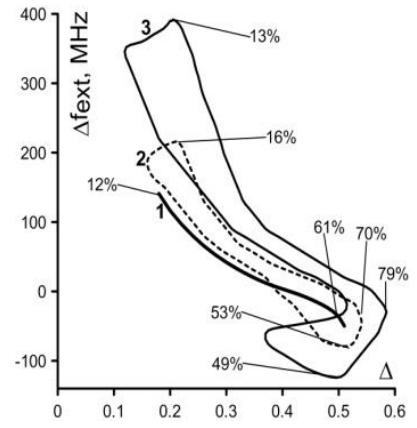

Fig. 2. Locking zones of 7-mode gyrotron with different level of external signal power at I $=50 \mathrm{~A}: 1$ ) free gyrotron, 2) external power is $1 \%$ of radiation power, 3 ) $5 \%$

\section{Frequency locking in the multimode gyrotron by a signal with varying frequency}

We study the frequency locking of the multimode gyrotron by an external signal with a variable frequency close to the operating mode frequency. The radiation frequency follows the frequency of the external signal when the difference between the external signal frequency and free oscillations frequency does not exceed the locking bandwidth and the locking time is smaller than the characteristic time of external signal frequency variations. When the first condition is violated, beats between free oscillations and external signal occur leading to decay into neighboring modes (fig. 3). If the locking time is 
longer than the characteristic time of external signal frequency variations, the radiation frequency does not track the variations of external signal frequency and oscillates in an essentially smaller interval.
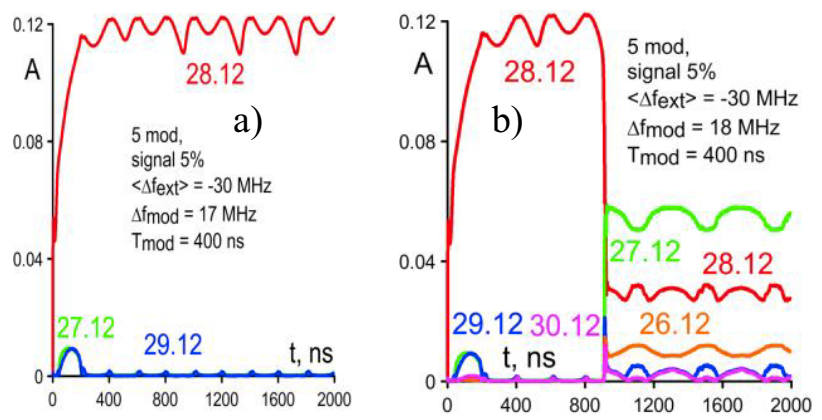

Fig. 3. Oscillograms of mode amplitudes in multimode gyrotron at a frequency of $170 \mathrm{GHz}, I=60 \mathrm{~A}, \Delta=0.58$, under influence of a signal with a frequency harmonically varying in time; $\left\langle\Delta f_{\text {ext }}\right\rangle$ is the difference between mean external frequency and 'cold' frequency of the operating mode, $T_{\text {mod }}$ is the period and $\Delta f_{\bmod }$ is the amplitude of external frequency modulation; locking bandwidth is $35 \mathrm{MHz}$; a) locking of operating mode frequency at $\Delta f_{\text {mod }}=17 \mathrm{MHz}$; b) decay of operating mode into parasitic ones at $\Delta f_{\bmod }=18 \mathrm{MHz}$

\section{Experimental results}

Locking of the gyrotron frequency by the signal of a magnetron was studied experimentally. In these experiments, the gyrotron represented an output section of a gyroklystron operating as an autooscillator at the frequency $35 \mathrm{GHz}$. The magnetron signal came into the gyrotron cavity through synthesized mode converter [7] (Fig. 4). Such a scheme permits to lock the frequency of megawatt power gyrotron unlike to the schemes used in previous experiments for low-power gyrotron with direct input of an external signal $[3,4]$. Dependence of the locking bandwidth on the power of the magnetron signal was determined (Fig. 5). Experimental data are in a good agreement with the results of numerical simulations.

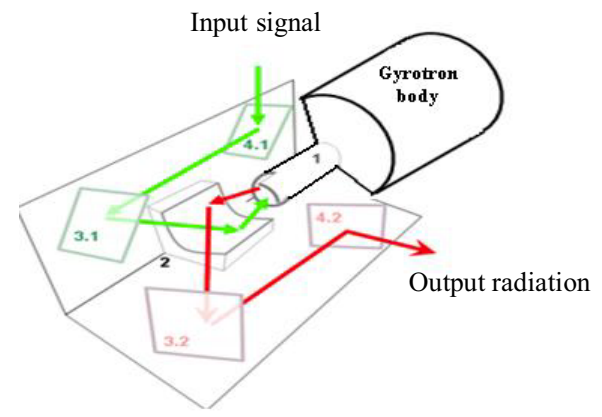

Fig. 4. Experimental setup scheme, 1 - launcher, 2 - quasiparabolic mirror; 3.1, 4.1 - matching input mirrors; 3.2, 4.2 matching output mirrors

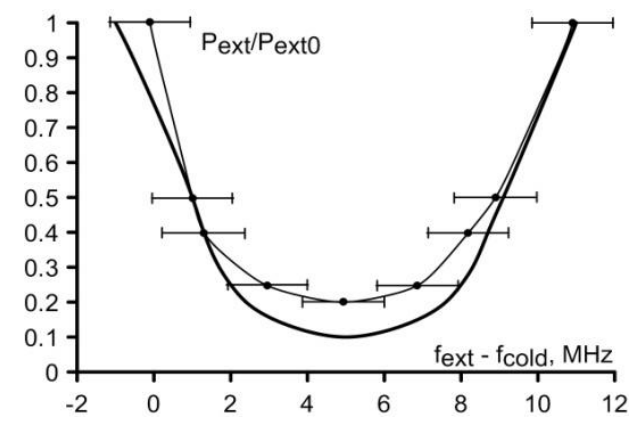

Fig. 5. Boundaries of locking band depending on the ratio between power of the magnetron signal and its maximum power. Thick curve corresponds to simulation, thin one corresponds to experiment. Maximum magnetron power level was 1/36 of gyrotron radiation power $(900 \mathrm{~kW})$

\section{Conclusion and acknowledgments}

Influence of external monochromatic or narrow-band wave is a promising method for frequency stabilization of gyrotron.

This work was supported by RFBR grant N 15-0201798A. We are grateful to A. N. Kuftin for useful discussions.

\section{References}

1. Ergakov V.S., Moiseev M.A. To the the theory of synchronization of CRM-monotron // Journal of Communications Technology and Electronics. 1978. N 12. P.25912599.

2. Nusinovich G.S. Introduction to the Physics of Gyrotrons. Baltimore: The Johns Hopkins University Press. 2004. $335 \mathrm{p}$.

3. Read M.E., Seeley R.,.Manheimer W.M. Observations of phase locking in a single-cavity gyrotron oscillator // IEEE Trans. On Plasma Science. 1985. V. PS-13, N.6. P.398-403.

4. Guo H., Hoppe D.J., Rodgers J., Perez R.M., Tate J.P., Conroy B.L., Granatstein V.L., Bhanii A.M., Latham P.E., Nusinovich G.S. Phase-Locking of a Second-Harmonic Gyrotron Oscillator Using a Quasi-Optical Circulator to Separate Injection and Output Signals // IEEE Trans. On Plasma Sci. 1995. V.23, N.5. P.822-832.

5. Ginzburg N.S., Glyavin M.Yu., Zavol'skii N.A., Zapevalov V.E., Moiseev M.A., Novozhilova Yu.V. A Proposal to Use Reflection with Delay for Achieving the Self-Modulation and Stochastic Regimes in Millimeter-Wave Gyrotrons // Technical Physics Letters. 1998. V. 24, Issue: 6. P. 436-438

6. Dumbrajs O., Idehara T., Watanabe S., Kimura A., Sasagawa H., Agusu L., Mitsudo S., Piosczyk B. Reflections in Gyrotrons With Axial Output. // IEEE Transactions on Plasma Science. 2004.V. 32, Issue 3. P.899-902.

7. Chirkov A.V., Denisov G.G., and Kuftin A.N. Perspective gyrotron with mode converter for co- and counter-rotation operating modes. // Applied Physics Letters 106, 263501 (2015); doi: $10.1063 / 1.4923269$. 